\title{
La formation tout au long de la vie
}

Les implications pour la politique éducative

\section{Abrar Hasan}

\section{CpenEdition}

\section{Journals}

Édition électronique

URL : http://journals.openedition.org/ries/3025

DOI : 10.4000/ries.3025

ISSN : 2261-4265

Éditeur

Centre international d'études pédagogiques

Édition imprimée

Date de publication : 1 décembre 1997

Pagination : 35-47

ISSN : $1254-4590$

Référence électronique

Abrar Hasan, "La formation tout au long de la vie », Revue internationale d'éducation de Sèvres [En ligne], 16 | Décembre 1997, mis en ligne le 07 juin 2013, consulté le 19 avril 2019. URL : http:// journals.openedition.org/ries/3025; DOI : 10.4000/ries.3025

Ce document a été généré automatiquement le 19 avril 2019

(c) Tous droits réservés 


\section{La formation tout au long de la vie}

Les implications pour la politique éducative

Abrar Hasan

\section{NOTE DE L'AUTEUR}

Le point de vue ici est celui de l'auteur et ne reflète pas nécessairement les points de vue de l'Organisation, ni de ses membres.

1 Le concept de formation tout au long de la vie est désormais largement reconnu par les gouvernements dans les pays de l'OCDE, les spécialistes de l'éducation, les employeurs, les syndicats et le public en général comme principe essentiel de la politique de l'éducation et de la formation ${ }^{1}$. Au cours des dernières années, trois des plus importantes organisations internationales, l'OCDE ${ }^{2}$, l'Union Européenne ${ }^{3}$ et l'UNESCO ${ }^{4}$ ont publié des rapports capitaux qui affirment ce principe et y souscrivent. À l'intérieur de l'OCDE même, des réunions entre les ministres de l'Éducation, les ministres de l'Emploi et le Conseil des ministres de l'OCDE ont accueilli ce concept avec enthousiasme. Il y a, cependant, un réel danger que ce concept ne devienne un terme vide de sens pour orienter la politique éducative puisqu'il implique des choses différentes pour des personnes différentes. L'objectif de cet article est de montrer que l'on peut donner un sens assez précis à ce concept et qu'il peut être une base utile pour orienter la politique de l'éducation et de la formation.

2 Bien sûr, l'expression «formation tout au long de la vie » n'est pas nouvelle. Dans le monde moderne, on la voit depuis au moins trois décennies, mais, récemment, la notion a pris un sens nouveau.

\section{L'évolution de cette notion}

3 L'origine de ce concept de «formation tout au long de la vie» se trouve dans des termes tels « l'éducation continue », «l'éducation permanente » et « l'éducation récurrente » ou en anglais life long education, recurrent education et continuing education ${ }^{5}$. Cette notion est 
apparue pour la première fois en anglais au cours des années vingt, associée à la tradition d'éducation pour adultes des pays nordiques, qui elle-même remonte au milieu du XIX siècle ${ }^{6}$. Malgré cette longue histoire, on a commencé à débattre de ce sujet un peu partout seulement après la Deuxième Guerre mondiale, et il n'a commencé à avoir une signification dans la politique à adopter qu'au début des années soixante.

Une conférence internationale sur l'éducation des adultes qui s'est tenue à Montréal, en 1960, sous l'égide de l'UNESCO, a cherché à intégrer l'éducation des adultes dans les systèmes éducatifs. Le document préparatoire à la conférence suivante (1965) formulait des propositions pour la mise en place de l'éducation tout au long de la vie ${ }^{7}$. Cependant, en 1969, lors d'un discours prononcé à la Conférence des ministres européens de l'Éducation à Versailles, le ministre de l'Éducation suédois, Olaf Palme, a lancé pour la première fois la notion distincte d'«éducation récurrente $»^{8} A u$ début des années soixante, cette notion a été popularisée sur le plan international par l'UNESCO ${ }^{9}$ l'OCDE ${ }^{10}$ et le Conseil de l'Europe ${ }^{11}$.

5 Ainsi, on s'est mis à employer l'expression « éducation tout au long de la vie » pour parler de l'éducation récurrente, au cours des années soixante-dix. On peut le noter dans des titres d'ouvrages publiés à cette période tel Recurrent Education: A strategy for Lifelong Learning et Recurrent Education and Lifelong Learning. Le principe de base de l'éducation récurrent e était que l'on devrait proposer l'éducation postscolaire de manière récurrente, pour faire en sorte qu'il y ait une alternance entre le travail et les études et que, tout au long de leur vie active, tous les individus aient effectivement des occasions de se former. Cette idée a été développée dans un rapport qui clarifie ce principe Clarifying Report, préparé par l'OCDE et présenté à la Conférence des ministres européens de l'Éducation qui s'est tenue à Berne (1973).

Ce terme était interprété soit de façon restrictive, soit dans une acception plus large. Dans son sens le plus étroit, « l'éducation récurrente » concerne la participation discontinue et périodique à des programmes d'éducation d'adultes dont la scolarité initiale avait été déficiente. Dans ce sens, on l'a considérée comme une alternative à l'allongement constant de la durée de l'éducation chez les jeunes. Dans son sens le plus large, il s'agit de gommer petit à petit les distinctions entre le bloc d'éducation obligatoire et la vie professionnelle ${ }^{12}$. Cette formule prône l'idée selon laquelle l'éducation récurrente sousentend implicitement une alternance entre l'éducation et d'autres activités. Cela concernait non seulement l'éducation après l'école, mais aussi à l'intérieur du système scolaire, et impliquait des changements importants dans les institutions sociopolitiques et économiques.

7 En pratique, c'est l'interprétation la plus étroite qui a dominé l'expression politique. Cependant, même dans ce contexte étroit, le succès était limité. Malgré une croissance considérable du domaine de la formation des adultes depuis 1960 et les déclarations répétées sur son importance par des gouvernements et d'autres organismes, le secteur est resté isolé et financièrement pauvre. L'aide supplémentaire des gouvernements a été surtout concentrée sur l'amélioration de la préparation au travail et, de bien des façons, l'éducation pour adultes est restée l'affaire des collectivités locales, financée par elles, plutôt qu'un secteur essentiel de la politique publique ${ }^{13}$. 


\section{La nouvelle notion et ses implications politiques}

8 Au cours des deux dernières décennies les développements ont fait ressortir un nouveau sens de la notion d'apprentissage tout au long de la vie. Cela ne se limite plus à l'éducation récurrente ou à l'éducation pour adultes. Il se réfère plutôt à l'activité d'apprendre qui se passe tout au long de la vie, depuis le berceau jusqu'à la mort. On peut considérer que quatre critères doivent être réunis dans une société qui apprend tout au long de la vie. Chaque individu, jeune ou vieux devrait se sentir motivé et équipé pour s'engager à apprendre d'une manière continue tout au long de sa vie, dans un cadre formel ou informel, institutionnalisé ou autre ; avoir l'occasion d'accéder à l'éducation permanente en bénéficiant d'incitations à la fois financières et sociales, pour saisir de telles occasions. Il est clair que la nouvelle notion de formation tout au long de la vie est très différente de l'éducation récurrente. Il s'agit d'un concept bien plus large qui englobe aussi bien l'éducation du jeune enfant que l'apprentissage chez des adultes plus âgés. Le vieil argument en faveur de l'éducation des adultes est inversé et on souligne le fait que de bonnes bases d'éducation sont tout aussi importantes pour les jeunes que pour les adultes. Il faut donc mettre l'éducation récurrente en perspective.

Comme le nouveau concept est très vaste et qu'il peut signifier des choses différentes pour des individus différents, les critiques disent que cela ne peut pas servir de base utile pour la politique de l'éducation. Cette section soutient que si le concept d'apprentissage tout au long de la vie est bien articulé, il fournit un cadre clair et unitaire pour le développement de la politique éducative. Les quatre critères identifiés ci-dessus peuvent être utilisés pour éclairer un certain nombre de questions politiques. En guise d'illustration, cette section examine l'éclairage qu'ils donnent en ce qui concerne: les objectifs, la structure, le contenu de la politique d'éducation, le financement de l'éducation et de la formation et, enfin, la répartition des rôles et des responsabilités entre les différents partenaires concernés, ceux qui fournissent l'éducation et ceux qui y participent. Dans chacun de ces cinq domaines, le principe d'apprendre tout au long de la vie offre une orientation spécifique à la politique d'éducation. Au-delà de ces illustrations spécifiques, cependant, l'importance majeure du concept réside dans le fait qu'il fournit un cadre complet pour la conception, le développement et la mise en place de la politique d'éducation, cadre qui, encore récemment, manquait cruellement dans le domaine de l'éducation.

\section{Les objectifs de la politique de l'éducation}

Cela fait des décennies, sinon des siècles, que l'on parle de la nature de la mission de l'éducation. Une série d'objectifs est très largement reconnue : encourager indépendance d'esprit et curiosité, aider au développement et à l'accomplissement de l'individu, le préparer à la vie professionnelle et à la citoyenneté, enrichir la vie sociale et culturelle, etc. Cependant, de plus en plus, se creuse un fossé entre ceux qui conçoivent l'éducation comme étant au service des besoins de l'économie et du marché du travail et ceux qui considèrent ces visées comme fondamentalement en conflit avec les autres objectifs de l'éducation. Le principe d'apprendre tout au long de la vie donne une perspective claire. Comme son nom l'indique, il implique qu'il faut considérer les objectifs éducatifs comme intégré, puisqu'ils concernent des phases différentes de la vie. Les quatre critères cités ci- 
dessus en sont la clé, puisqu'ils recouvrent plusieurs des missions mentionnées. De ce point de vue, chacun des objectifs énumérés ci-dessus a sa place et doit être pris en compte. Le débat sur la supériorité d'une série d'objectifs sur une autre est superflu dans ce contexte. Ce qui est pertinent, c'est de savoir comment on peut utiliser leurs points de convergence pour mieux atteindre chacun d'entre eux.

11 Le nouveau concept de formation tout au long de la vie fait penser que ces tensions et ces débats ont été exagérés. On doit estimer que l'éducation et la formation font partie d'un système, qu'elles sont ancrées profondément dans l'évolution de la dynamique de la société et qu'elles contribuent à ces développements tout en étant influencées par eux. Ce point de vue est conforté par la nouvelle convergence que l'on peut observer entre les différents buts de l'éducation. Un récent sondage, conduit dans douze pays de l'OCDE, montre que le public, en général, attend de l'enseignement scolaire qu'il dote les élèves de qualités telles que la confiance en soi, des compétences et des connaissances nécessaires pour trouver un travail et de la capacité de vivre parmi des populations de cultures et de milieux différents. Le public attache encore plus d'importance à ces qualités qu'à l'enseignement de matières spécifiques ${ }^{14}$. Le monde du travail est en train de définir toute une gamme de compétences transversales - la communication, des compétences multilingues, la créativité, le travail d'équipe, la résolution de problèmes, une familiarité avec les nouvelles technologies - qui sont d'une importance de plus en plus grande pour obtenir un emploi et avoir la flexibilité requise pour s'adapter aux besoins changeants du travail. Certaines de ces compétences sont tout aussi importantes pour toutes les missions de l'éducation: promotion d'une bonne citoyenneté, accomplissement personnel, esprit indépendant et curieux, conscience des droits sociaux et des responsabilités et préparation au travail. La nécessité d'une éducation la plus généraliste possible semble être valorisée de plus en plus par tous ceux qui sont impliqués dans l'éducation : les individus, les familles, les spécialistes de l'éducation, les entreprises, les gouvernements et la société dans son ensemble. La formation tout au long de la vie, dans sa nouvelle acception, fournit un cadre pour la conceptualisation et un principe directeur en fonction duquel divers objectifs et missions de l'éducation et de la formation se renforcent mutuellement.

\section{La structure et la composition de l'offre d'éducation}

L'approche qui consiste à considérer que la formation se déroule tout au long de la vie, de façon systémique, la mise en œuvre de l'éducation et de toutes les occasions d'apprendre, prend aussi bien en compte l'éducation formelle et informelle que les apprentissages dans un cadre non structuré. La perspective d'apprendre tout au long de la vie pose un certain nombre de questions qui, autrement, n'auraient pas été soulevées. Est-ce que l'offre d'éducation correspond bien aux besoins? Dans cette perspective, est-ce que les ressources attribuées aux différents secteurs, traditionnels ou non, sont appropriées? Existe-t-il des liens et des passerelles satisfaisants entre les occasions d'apprendre dans ces cadres différents? Est-ce que le niveau et la qualité sont maintenus par les diverses formes de l'offre et est-ce que les acquis sont certifiés et reconnus de façon satisfaisante? Ce point de vue systémique donne une responsabilité particulière à tous ceux qui offrent de la formation : celle de ne pas considérer leurs offres comme des systèmes fermés mais, au contraire, ouverts et reliés à d'autres sources d'éducation. C'est une caractéristique essentielle de l'éducation tout au long de la vie. Cela nécessite une grande capacité 
d'ouverture de la part des différents secteurs qui offrent de la formation et - de la part des acteurs concernés. Il leur faut avoir la volonté de coopérer avec d'autres « fournisseurs » d'éducation et de répondre aux changements rapide de la demande.

\section{Contenu, qualité et pertinence}

Les quatre critères mentionnés ci-dessus impliquent une nouvelle perspective dans l'évaluation du contenu de l'éducation. L'activité d'apprentissage doit être évaluée de façon dynamique - elle devrait non seulement générer de nouvelles connaissances, mais préparer et motiver les individus à poursuivre leur apprentissage. Un élément clé de la motivation vient du degré de pertinence de ce que l'on apprend, ce qui renforce le rôle de l'apprenant dans la définition de la pertinence. Le concept de formation tout au long de la vie met très nettement l'accent sur la promotion de l'auto apprentissage. Une autre question relève de la pédagogie : une bonne proportion de ceux qui quittent le système scolaire tôt ne trouve pas motivantes les approches pédagogiques suivies par les écoles. Beaucoup d'entre eux préféreraient une orientation plus pratique des programmes. Les programmes scolaires existants ignorent les compétences interdisciplinaires, alors qu'on a souligné leur importance ci-dessus. Elles ne prennent pas en considération non plus les élèves qui apprendraient mieux avec davantage de travail pratique ou de façon expérientielle. La question est aussi de savoir quelle pédagogie (ou andragogie) est pertinente pour des adultes. Les études ont montré que certains adultes ayant un faible niveau scolaire de départ avaient un souvenir désagréable des approches pédagogiques traditionnelles et scolaires. Dans l'optique d'apprendre tout au long de la vie, il serait important de développer de nouvelles méthodes d'enseignement et d'apprentissage qui conviennent à des groupes spécifiques d'apprenants.

\section{Financement}

14 L'approche actuelle en terme de coûts et de financement est, en général, sectorielle. Elle ne prend pas en compte les ressources qui concernent l'apprentissage informel, ni les bénéfices que l'individu et la société pourraient en tirer. Apprendre toute la vie nécessite une approche systémique des ressources consacrées aux diverses composantes de l'éducation formelle ou informelle. L'évaluation des coûts et bénéfices de l'éducation et de la formation doit être calculée en prenant en compte les ressources consacrées par la société pendant toute la vie d'un individu. L'approche financière doit englober les bénéfices, tout au long de la vie, pour l'individu comme pour la société.

\section{Rôles et responsabilités}

15 Il est clair que, vue l'étendue des activités qui entrent dans le cadre de l'éducation permanente, un grand nombre de participants et d'intervenants se partagent la responsabilité de l'offre de formation. Les stratégies employées pour l'éducation permanente soulignent l'importance de la coopération entre les différents acteurs. Il peut en résulter deux formes d'intégration. Sur le plan vertical, les différents secteurs de l'offre doivent former un ensemble cohérent: l'accès des adultes à la formation doit s'intégrer à la formation initiale. Sur le plan horizontal, éducation et formation doivent être coordonnées avec les autres politiques. Par exemple, combattre l'indigence de 
réduction, au niveau des jeunes enfants, exige des partenariats avec les services sociaux, la santé, le logement et les agences de l'emploi. Reconnaissant ce besoin, les ministres de l'Emploi de l'OCDE ont réclamé une réunion commune avec les ministres de l'Éducation et des Finances ${ }^{15}$, lors d'une réunion récente à Paris.

Une politique intégrée nécessite des partenariats entre le gouvernement et les différents acteurs. L'éducation des jeunes enfants, par exemple, nécessite une coordination avec des organismes volontaires au niveau local, régional et national. Pour les adultes, les gouvernements auraient besoin de développer de nouveaux partenariats avec les partenaires sociaux, pour encourager la formation sur le lieu de travail. Ces partenariats sont souvent plus productifs dans un cadre régional ou local. En général, ce sont les gouvernements qui sont dans la meilleure position pour fixer le cadre de la politique et pour développer les systèmes et les réseaux que les individus peuvent utiliser pour apprendre. Dans ce cadre, ils peuvent adopter un rôle stratégique et faire en sorte que les partenariats entre gouvernement, partenaires sociaux et intervenants se développent. Le rôle essentiel du gouvernement dans la mise en place de ce cadre est de fournir les informations nécessaires à tous ceux qui prennent des décisions, ainsi qu'aux individus, aux familles, aux entreprises, aux partenaires sociaux et aux organisations communautaires.

\section{Raison d'être et défis}

17 Il est évident que fixer comme objectif l'éducation tout au long de la vie pour tous est ambitieux. On peut se poser la question de savoir s'il s'agit d'un objectif viable dans le long terme. Si l'on prend en considération la raison d'être qui sous-tend le soutien très large pour ce concept, on peut conclure que les pays de l'OCDE ne peuvent pas se permettre de ne pas soutenir les stratégies d'une formation tout au long de la vie pour tous.

La raison d'être de l'éducation tout au long de la vie réside dans les changements structuraux qui ont balayé les pays de l'OCDE depuis trois décennies ou plus. Des changements dans les domaines de la technologie, de la globalisation, de la déréglementation des marchés, de la démographie et dans de nouvelles économies émergeantes sont tous bien connus. Ils ont lancé de nouveaux défis, tout comme ils ont apporté de nouvelles ouvertures. Ces pressions ont considérablement modifié la composition des capacités et des compétences exigées, ainsi que la nature de l'offre, accroissant leur diversité respective. Sur le plan social, on risque d'avoir une nouvelle polarisation. Les forces économiques et sociales se conjuguent pour fournir une solide raison d'être à l'éducation tout au long de la vie.

La révolution dans le domaine de l'information a davantage mis l'accent sur les « sociétés apprenantes » et les connaissances de base deviennent un élément de plus en plus déterminant pour le progrès social et économique. Produits et compétences deviennent plus rapidement obsolètes, au fur et à mesure que la vitesse d'introduction de nouveaux produits et services s'accélère. Les sociétés industrielles avancées, en particulier, vivent une dépendance croissante à l'égard de la création et du bouleversement des connaissances, des informations et des idées. Les caractéristiques de cette société où s'intensifient connaissances et informations concernent les adultes d'un âge mûr tout aussi bien que les enfants et les jeunes. Les pays et les régions qui n'encouragent pas la 
société apprenante et les individus qui n'y participent pas, sont de plus en plus désavantagés et ont toutes les chances de rester à la traîne, non seulement sur le plan national, mais aussi dans le contexte international.

Le vieillissement de la population dans les pays industrialisés, les améliorations sur le plan de la santé et des loisirs partout dans le monde ont fait en sorte qu'il est possible de prolonger la vie active, tout comme la période d'éducation initiale a été prolongée pour les jeunes. L'activité économique d'un individu sur le marché du travail est de plus en plus concentrée au milieu de sa vie de travail, entre une période prolongée d'éducation au départ et une période de retraite plus longue à l'autre bout de la vie. Le poids des dépenses qui incombent au groupe plus restreint des actifs du «milieu» est plus important, et il y a donc des raisons économiques et sociales pour allier travail de production et apprentissage, pour ne pas trop réduire les périodes d'activité économique productive.

21 La nature des compétences demandées change et le seuil des compétences s'élève. La demande des entreprises d'une plus grande flexibilité a rendu le travail plus précaire et on utilise plus souvent des formes de travail qui ne sont plus dans les normes. Il y a une tendance vers des contrats de travail plus courts, qui reflètent un marché des produits plus volatile et des cycles de production courts. Les individus changent plus souvent de travail au cours de leur vie que par le passé. Le modèle primaire de gestion qui est en vogue depuis la Deuxième Guerre mondiale, que l'on appelle taylorisme ou fordisme, subit des changements. Ce modèle avait tendance à abaisser les compétences exigées des travailleurs. Plus simplement, selon cette approche, une grande majorité de travailleurs, sans grande spécialisation, accomplissaient des tâches étroitement définies, en étant encadrés par un petit groupe de travailleurs spécialisés qui devaient résoudre les problèmes et innover. On se rend de plus en plus compte que la qualité et l'engagement des travailleurs est une composante essentielle de la compétitivité de l'entreprise. En même temps, il est clair qu'un fossé s'est creusé entre le type de capacités exigées sur le lieu de travail et ce que les institutions éducatives fournissent. Les employeurs apprécient davantage un personnel capable d'appliquer ce qu'il sait et qui résout facilement les problèmes. Les compétences de communication et le travail d'équipe priment pour eux.

22 Le chômage a eu tendance à augmenter dans la plupart des pays de l'OCDE, depuis le milieu des années soixante-dix. Il invite de façon impérative à adopter ce nouveau concept de formation tout au long de la vie. Les taux de chômage des jeunes adultes sont très élevés, en général une fois et demie le taux des adultes, parfois deux fois ce taux ; la proportion est en augmentation dans presque tous les pays de l'OCDE depuis 1979. Cette augmentation est inquiétante en elle-même, mais elle l'est d'autant plus qu'elle arrive à un moment où la proportion de jeunes adultes dans la population de l'OCDE baisse. On estime que des stratégies pour apprendre tout au long de la vie sont un atout considérable pour améliorer les chances d'avoir un emploi tout au long d'une vie.

Ce nouvel accent sur l'éducation permanente ne vient pas uniquement des forces économiques. Il y a des facteurs sociaux tout aussi puissants. Toute l'évidence semblerait suggérer que l'on risque de voir le fossé se creuser entre ceux qui ont des connaissances et ceux qui n'en ont pas, ce qui peut menacer le fondement même de la démocratie. Les occasions de se former plus tard au cours de la vie dépendent beaucoup des qualifications déjà acquises à l'arrivée sur le marché du travail. Le fossé dans les occasions de se former se creuse avec l'âge. De même, les formations ouvertes aux travailleurs dans les petites entreprises sont nettement moins nombreuses que celles offertes à des travailleurs dans 
les grandes entreprises. Il y a également une grande disparité entre les opportunités de formation proposées aux chômeurs et aux groupes démunis de la société et celles proposées à ceux qui ont un travail. Les formations proposées diminuent aussi considérablement après 45 ans. Par conséquent, l'écart des salaires entre ceux qui suivent une éducation supérieure et ceux qui n'y arrivent pas est grand et s'accroît au cours de la vie. Il semblerait que cet écart se soit creusé entre les années soixante-dix et les années quatre-vingt-dix. On considère qu'avoir des stratégies pour une formation tout au long de la vie est nécessaire pour lutter contre l'exclusion et la marginalité. Les activités éducatives sont une source d'implication et de participation particulièrement importante au vu des forces qui mettent la cohésion sociale en danger.

24 Toutes ces remarques sont corroborées par les informations sur les salaires et les taux de chômage. Malgré une grande augmentation du coût des inscriptions à l'université, les gains d'une éducation universitaire restent élevés. Quand on compare les salaires des adultes avec et sans éducation supérieure, les données montrent une grande différence dans les salaires. Ceux qui ont une éducation plus poussée y gagnent aussi d'une autre façon. Ils ont un plus grand nombre d'occasions de suivre une formation formelle que les autres employés. Aussi, le taux de chômage est-il plus faible parmi ceux qui ont des diplômes universitaires que chez les actifs qui n'en ont pas. Les données concernant les créations d'emploi montrent une forte croissance au profit de ceux qui ont de très bonnes qualifications. Pour chacun des huit pays, le taux de croissance des emplois de bureau est positif, que ce soit pour des emplois très spécialisés ou non, alors que le taux est en général négatif pour les ouvriers, qu'ils soient spécialisés ou non.

La conclusion générale est que des impératifs économiques, personnels et sociaux se combinent pour souligner le fait qu'apprendre tout au long d'une vie n'est pas un luxe, mais est plutôt en train de devenir très rapidement une nécessité. La prospérité économique future, la cohésion politique et sociale et la création de sociétés réellement démocratiques, où chacun participe à la vie publique, dépend totalement de la qualité de l'éducation et de la formation.

\section{Les défis}

Des progrès considérables ont été réalisés dans les pays industrialisés, au cours des trois dernières décennies, pour dispenser l'éducation et la formation. La plupart des pays industrialisés ont réussi à mettre en place une éducation secondaire universelle. Ce qui est recherché, c'est l'éducation de masse post-bac, c'est-à-dire non seulement l'université, mais aussi les autres formes de l'éducation technique et générale. Par conséquent, l'éducation tout au long de la vie est déjà une réalité pour certains des pays de l'OCDE. Cependant, des défauts importants demeurent sur les plans de la qualité et de la quantité.

Bien que l'éducation des jeunes enfants soit bien établie dans certains pays de l'OCDE, il reste encore à en améliorer l'accès et la qualité. Au niveau secondaire, le taux de scolarisation à un an de la fin de la scolarité est proche de $100 \%$ dans la plupart des pays, mais il baisse de manière significative au cours de la dernière année d'école obligatoire. Ce qui est plus grave, c'est qu'une minorité importante d'une classe d'âge continue à quitter l'école sans diplôme valable. Le fort taux de scolarisation masque pourtant une diversité considérable dans les performances des élèves, ce qui indique des déficiences dans les compétences de base. Par exemple, le fossé entre ceux qui atteignent le niveau moyen en lecture et ceux qui sont largement au-dessus ou largement en-dessous 
représente entre deux et quatre années de scolarité dans certains pays. L'étude récente, International Adult Literacy Survey (IALS), une enquête sur la lecture chez les adultes ${ }^{16}$, montre que dans plusieurs pays, jusqu'à $35 \%$ des jeunes adultes (âgés de 16 à 29 ans) n'atteignent que les deux niveaux les plus faibles en lecture. La phase de transition entre école et travail devient de plus en plus longue : les plus bas salaires et un taux de chômage plus élevé vont de pair avec les résultats scolaires.

L'enquête sur la lecture a aussi montré de sérieux défauts dans les capacités et les compétences des adultes en mesure de travailler. L'enquête a évalué toute une gamme de compétences dont la lecture de textes et de documents - et les compétences numériques. La révélation majeure et peu rassurante est qu'un quart des adultes ou plus, dans la plupart des pays, ne réussissent à atteindre que le niveau le plus bas des compétences.

On peut remarquer que le modèle varie considérablement selon les pays. Au cours d'une période de référence de douze mois, à partir des données provenant de sept pays de l'OCDE, entre 34 et 40 pour cent des adultes employés âgés de 25 à 64 ans ont indiqué avoir suivi un apprentissage lié à leur travail. Quand on se réfère à une période d'un mois, le taux de participation varie de 1 à 15 pour cent. Quand on compare ce taux à celui des chômeurs, on voit que le taux pour ces derniers est beaucoup plus bas. Les données de l'IALS montrent que, dans l'ensemble, le taux de participation varie de 34 à $53 \%$ pour le groupe d'âge qui va de 15 à 64 ans. La participation est généralement plus faible pour les femmes que pour les hommes et baisse considérablement au-delà de 45 ans. La conclusion principale est qu'une majorité d'adultes dans les pays industrialisés ne participent pas à la formation tout au long de la vie.

Si l'on considère que l'objectif d'une éducation tout au long de la vie pour tous permet de conduire chaque individu au moins au niveau de l'enseignement secondaire, il faudrait toucher quelques soixante-seize millions d'adultes dans le groupe des 25 à 64 ans.

Si l'on choisi pour objectif les critères de l'IALS d'atteindre au moins le niveau II en lecture, rien qu'aux États-Unis, quelques soixante-cinq millions de personnes auraient besoin de se former dans le groupe d'âge allant de 26 à 65 ans. On peut estimer qu'en gros, le coût nécessaire pour toucher ce groupe de la population se situerait entre 1,3\% et 4,4\% du PNB pour les pays de l'OCDE.

Les points essentiels soulevés ci-dessus doivent être résumés, avant que de conclure par quelques remarques. La notion d'éducation tout au long de la vie, dans son acception depuis le début des années soixante-dix, se limitait à donner une seconde chance aux adultes qui avaient raté leur scolarité initiale. Cette stratégie n'a connu qu'un succès limité. Des changements depuis les années soixante-dix, surtout ceux qui concernent la globalisation, la technologie, la démographie et la structure de l'emploi, ont généré une diversité de plus en plus grande dans les demandes de formation et les dispositifs d'apprentissage.

33 Ces changements dans l'offre et la demande font peser de nouvelles contraintes sur le système. Le nouveau concept d'éducation tout au long de la vie qui prône l'apprentissage du « berceau jusqu'à la mort », est en rupture par rapport à l'ancien concept. Ce concept élargi est accepté très largement comme une réponse à des pressions économiques et 
sociales importantes. En même temps, le concept est critiqué comme étant trop large pour donner une orientation significative à la politique éducative.

Dans cet article, ce qui est suggéré c'est que le nouveau concept fournit un cadre systémique pouvant servir à mieux formuler et mettre en place une politique d'éducation et de formation. Des exemples de ces approches dans différents domaines spécifiques illustrent la discussion en ce qui concerne les objectifs, les structures, le contenu, le financement et la conduite de la politique de l'éducation. Adopter le cadre de l'éducation tout au long de la vie implique une nouvelle donne en ce qui concerne ces différents domaines.

L'éducation tout au long de la vie est déjà une réalité pour une petite proportion des pays de l'OCDE. Cependant, étendre l'application de ce concept à « tous », objectif à long terme soutenu par les ministres de l'Éducation de l'OCDE lors de leur réunion de janvier 1996, est un but ambitieux qui ne peut être atteint que progressivement. La dimension quantitative de l'éducation n'est qu'un des éléments; des changements majeurs sont nécessaires quant à la qualité et la pertinence avec lesquelles elle est dispensée. La mise en place de stratégies pour une éducation tout au long de la vie demande un effort de la part d'un grand nombre d'acteurs. Il faut repenser le rôle et les responsabilités du gouvernement et d'autres partenaires. Les ministères concernés doivent se réunir et mieux coopérer, pour éviter que certains éléments de la stratégie ne leur échappent.

La nouvelle conceptualisation de l'apprentissage au cours de la vie n'est pas une simple question de présentation. C'est une toute autre façon de voir les problèmes liés à l'éducation et à la formation. Plutôt que de traiter comme dichotomique l'opposition entre l'éducation formelle et informelle, entre l'éducation institutionnelle et non institutionnelle et plutôt que d'opposer ce que l'on apprend au départ à ce que l'on apprend par la suite, il faut voir l'éducation et la formation comme un ensemble. Les questions les plus importantes sont de savoir si le système, considéré comme un tout, va dans la bonne direction pour accomplir ses missions d'éducation, si les ressources financières aussi bien qu'humaines sont suffisantes et s'il fonctionne de manière cohérente.

Le thème de la cohésion sociale devient particulièrement important. La nouvelle vision de l'éducation et de la formation tout au long de la vie accorde une attention plus grande au rôle de l'éducation et de la formation en tant que politique de promotion de la cohésion sociale. Le secteur de l'éducation doit être plus clairement traité comme faisant partie de la dynamique sociale. Le fait que l'on peut apprendre dans des endroits différents et, pas seulement dans des établissements scolaires, exerce une pression sur ces établissements pour qu'ils s'ouvrent davantage et soient plus réceptifs à la diversité des besoins. Même si cela crée de nouvelles tensions, cela ouvre aussi d'immenses perspectives. Malgré la progression rapide du nombre des inscrits, y compris au niveau post-bac, les institutions éducatives n'ont pas fait beaucoup de progrès dans l'ouverture de nouvelles propositions de formation pour les individus les plus défavorisés de la société. Les stratifications sociales ont même été reproduites, en grande partie, dans le secteur post-baccalauréat ${ }^{17}$. Ainsi promouvoir la cohésion sociale est un objectif important dans l'approche éducative de la formation tout au long de la vie, souligné encore par le rajout de « pour tous » dans l'intitulé de la réunion des ministres de l'Éducation de l'OCDE de 1996. 


\section{NOTES}

1. Comme on l'expliquera par la suite, la formation professionnelle fait partie de l'éducation permanente. Cependant, pour être bref, on parlera de la politique de l'éducation pour couvrir toute une gamme de politique d'éducation et de formation

2. Lifelong Learning for All, Meeting of Education Committee at Ministerial Level, 16-17 January, Paris, OCDE, 1996.

3. Commission européenne, White Paper on Education and Training: Teaching and Learning - Towards the Learning Society, Luxembourg, 1995.

4. Unesco, Report of the Commission, International Commission on Education for the Twenty-first Century, Paris, Unesco, 1996.

5. D. Kaller, «Recurrent Education and Lifelong Learning: Definitions and Distinctions », in Schuller and Pegarry (eds.), London, Kogan Page, 1979 ; A. Hasan, « Lifefong Learning », in International Encyclopedia of Adult Education and Training, Oxford, Elsevier Science, 1996.

6. P. Sutton, «Lifelong and Continuing Education", Internatinal Encyclopedia of Education, 2nd Edition, Oxford, Pergamon Press, p. 3416-3422 ;

7. P. Lengrand, Éducation permanente, Paris, Unesco, 1965.

8. V. Houghton, Recurrent Education - An Alternative Future, Milton Keynes, The Open University, 1974; P. Jarvis, Adult an Continuing Education, New York, Croom Helm, Beekenham, 1983 ; T. Schuller and J. Megarry, Recurrent Education and Lifelong Learning, World Year Book of Education (eds.), London, Kogan Page, 1979.

9. UNESCO, Learning to be (le rapport Faure), Paris, Unesco, 1972.

10. OCDE, Recurrent Education. A strategy for Lifelong Learning, Paris, OCDE, 1973 ; OCDE/ CERI, Recurrent Education. Trends and Issues, Paris, OCDE, 1975; OCDE, Learning Opportunities for Adults, vol. 1-5, Paris, OCDE, 1977-1981; OCDE, The Lifelong Learner in the 1990s, document CERI/CD (91) 16, Paris, OCDE, 1991 ; OCDE, Adult Literacy and Economic Performance, Paris, OCDE, 1992.

11. Conseil de l'Europe, Permanent Education (rapport final), Strasbourg, Conseil de l'Europe, 1978.

12. M. Jourdan, Recurrent Education in Western Europe, Windsor, NFER, Nelson, 1981.

13. G. Papadopoulos, Education 1960-1990: The OECD Perspective, Paris, OCDE 1994.

14. OCDE, Prepared for life, Paris, OCDE, 1977.

15. OCDE, Communique, Meeting of Employement, Labour and Social Affairs, Committee at Ministerial Level, Paris 14-15 October 1997.

16. OECD and Statistics Canada, Literacy, Economy and Society, Paris, OCDE, 1995.

17. OCDE (à paraitre), Comparative Report on the Thematic Review of First Years of Tertiary Education. 


\section{RÉSUMÉS}

La reconnaissance du concept de formation tout au long de la vie constitue désormais un acquis pour les pays de l'OCDE. Avant d'en arriver à ce consensus, les évolutions ont été multiformes. L'auteur en décrit les diverses phases et identifie les points de rencontre entre les diverses acceptions. Il met en garde aussi contre le risque inhérent à tout consensus lorsque la tendance au rapprochement vide de sens ce qui devrait porter l'innovation. Pour que le concept soit innovant et porteur d'espoir, l'auteur inventorie les conséquences de sa mise en pratique sur les politiques d'éducation.

\section{INDEX}

Index géographique : Europe, Pays de l'OCDE

Mots-clés : formation tout au long de la vie, politique éducative

\section{AUTEUR}

\section{ABRAR HASAN}

Chef de la division de l'éducation et de la formation, OCDE, Paris, France 